\title{
Identity elements in tRNA-mediated transcription antitermination: implication of tRNA D- and T-arms in mRNA recognition
}

\author{
Maarten van de Guchte, S. Dusko Ehrlich and Alain Chopin
}

Laboratoire de Génétique Microbienne, Institut National de la Recherche Agronomique, 78352 Jouy-en-Josas Cedex France
Author for correspondence: Maarten van de Guchte. Tel: +3313465 25 28. Fax: +33134652521. e-mail: guchte@biotec.jouy.inra.fr

\begin{abstract}
tRNA-mediated transcription antitermination has been shown to control the expression of several amino acid biosynthesis operons and aminoacyl-tRNAsynthetase-encoding genes in Gram-positive bacteria. A model originally put forward by Grundy \& Henkin describes the conserved structural features of the leader sequences of these operons and genes. Two sequences of 3 and $4 \mathbf{n t}$, respectively, take a central position in this model and are thought to be responsible for the binding of the system-specific uncharged tRNA, an interaction which would stabilize the antiterminator conformation of the leader. Here a further evolution of this model is presented based on an analysis of trp regulation in Lactococcus lactis in which a function is assigned to hitherto unexplained conserved structures in the leader sequence. It is postulated that the mRNA-tRNA interaction involves various parts of the tRNA in addition to the anticodon and the acceptor in the original model and that these additional interactions contribute to the recognition of a specific tRNA, and hence to the specificity and efficacy of the regulatory response.
\end{abstract}

Keywords: gene regulation, T-box, tRNA, Lactococcus lactis

\section{INTRODUCTION}

A transcription antitermination mechanism which has received considerable attention during the last few years, employs a direct interaction between an mRNA and a specific tRNA to stabilize the antitermination conformation of the nascent mRNA transcript. By its nature, this mechanism of antitermination is especially apt to regulate the biosynthesis and use of amino acids. It has been found or hypothesized to govern the regulation of several amino acid biosynthesis operons and aminoacyltRNA-synthetase encoding genes, in a number of Grampositive bacteria.

The non-translated leader transcripts of these genes or operons share strikingly similar secondary structures in which the specifier codon occupies a crucial, conserved position (Fig. 1; Grundy \& Henkin, 1993; Chopin et al., 1998; Luo et al., 1998). This codon corresponds to the amino acid which the operon is responsible for the biosynthesis of, or in the case of aminoacyl-tRNAsynthetase-encoding genes, to the amino acid that is coupled to a tRNA by that particular synthetase, and is thought to bind to the corresponding tRNA in a

Abbreviation: CDM, chemically defined medium. codon-anticodon interaction. A second conserved position in the secondary structure is reserved for the antiacceptor which is situated in the actual terminator and is complementary to the four $3^{\prime}$ terminal nucleotides of the acceptor arm of the tRNA to which this sequence is thought to bind (Grundy \& Henkin, 1993; Grundy et al., 1994; Van de Guchte et al., 1998). The latter interaction explains the regulatory potential of this system. An uncharged tRNA can bind to the leader transcript, maintain the antiterminator conformation and thus promote the expression of the operon or gene. A tRNA that is charged with an amino acid on the acceptor arm, however, would not be able to bind and stabilize the antiterminator in the leader transcript. The latter adopts the energetically more favourable terminator conformation and expression of the operon or gene is suppressed.

We have presented evidence in support of this transcription antitermination model for the tryptophandependent regulation of the Lactococcus lactis trp operon (Van de Guchte et al., 1998). We showed for several specifier mutants that transcript elongation could be induced by the absence of the newly specified amino acid from the culture medium. The new response was much lower than the response to tryptophan depletion 


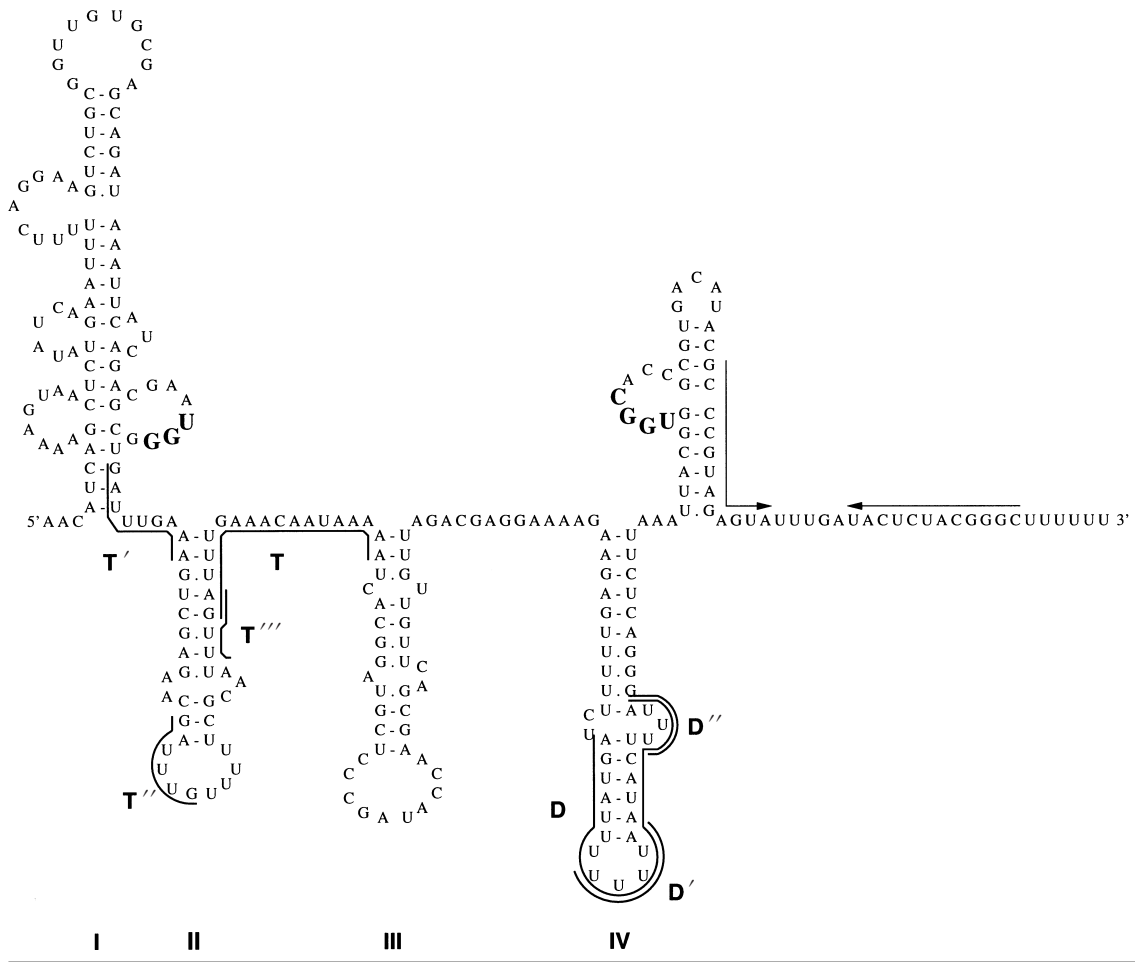

Fig. 1. Model of the antitermination conformation of the lactococcal trp leader. Arrows indicate the mutually exclusive transcription terminator (Bardowski et al., 1992; Frenkiel et al., 1998). Stem-loop structures I, II, III and IV are conserved in all T-box leaders (Rollins et al., 1997; Chopin et al., 1998). The specifier codon (UGG, in stem-loop structure I) and anti-acceptor (UGGC in the antiterminator) are indicated in bold type. T, Line indicates sequence complementary to the T- and extra arms of tRNA ${ }^{\text {Trp }}$ as presented in Fig. 4(a); $\mathrm{T}^{\prime}, \mathrm{T}^{\prime \prime}$ and $\mathrm{T}^{\prime \prime \prime}$, lines indicate partial repeats of the same sequence that are complementary to the tRNA T-loop; $D$, line indicates sequence complementary to the D-arm of tRNA ${ }^{\text {Trp }}$ as presented in Fig. 4(a); $D^{\prime}$ and $D^{\prime \prime}$, short lines indicate sequences complementary to the tRNA D-loop.

in the wild-type system, however, while, as became evident especially under conditions of overexpression of tRNA ${ }^{\text {Trp }}$, tryptophan depletion could still induce a low level of transcript elongation in these mutants.

These results showed that when acceptor and antiacceptor are compatible, specifier codon-anticodon complementarity is neither sufficient for an efficient response to occur nor strictly indispensable for a low level response, and consequently suggested the existence of additional means of recognition between the trp leader transcript and tRNA ${ }^{\text {Trp }}$. Here we present experimental results that strongly suggest the implication of the tRNA D- and T-arms in the recognition of conserved stem-loop structures in the trp leader transcript.

\section{METHODS}

Bacterial strains, plasmids and media. The bacterial strains and plasmids used in this study are listed in Table 1. Escherichia coli was grown in LB broth (Miller, 1972) or on LB solidified with $1.5 \%$ agar. L. lactis was grown in glucose M17 broth (Terzaghi \& Sandine, 1975) to which sucrose $(0 \cdot 5 \mathrm{M})$ was added to osmotically stabilize electroporated cells or on glucose M17 solidified with $1.5 \%$ agar.

RNA was extracted from L. lactis grown in chemically defined medium (CDM; Raya et al., 1998). When present, tryptophan, asparagine, cysteine, alanine, phenylalanine and tyrosine were used at $100 \mu \mathrm{g} \mathrm{ml}^{-1}$. Erythromycin was added where appropriate to $2 \cdot 5-5 \mu \mathrm{g} \mathrm{ml}^{-1}$ for L. lactis. Ampicillin was used at $150 \mu \mathrm{g} \mathrm{ml}^{-1}$ for E. coli.

DNA manipulations and transformation. E. coli and L. lactis were transformed by electroporation (Dower et al., 1988; Holo \& Ness, 1995). Plasmid DNA was isolated by the method of Birnboim \& Doly (1979). Restriction enzymes and
T4 DNA ligase were purchased from Boehringer or New England Biolabs and used according to the instructions of the suppliers. Taq DNA polymerase and Vent DNA polymerase for use in PCR were purchased from Promega and New England Biolabs, respectively. Polynucleotide kinase was purchased from New England Biolabs. PCR-mediated DNA amplification was performed using a Perkin Elmer Cetus DNA thermal cycler 2400 or 9600 . DNA sequence analysis was performed using an Applied Biosystems 373 automated DNA sequencer. Oligonucleotides were synthesized using a Beckman Oligo 1000M DNA synthesizer.

PCR-directed mutagenesis and overexpression of tRNAs. Mutations in the lactococcal $t r p$ leader region were generated by PCR using mutagenic primers as described previously (Van de Guchte et al., 1998). Mutated leader fragments (EcoRIHindIII) were subsequently cloned in the delivery vector pIL931 and integrated upstream of the E. coli lacZ gene in $L$. lactis IL56012 to reconstitute a functional trpE-lacZ fusion on the chromosome. The fusion is located within the his operon, which is transcriptionally silent in the presence of histidine in the culture medium (Delorme et al., 1999). Recombinant tRNA sequences were generated by PCR using two partially overlapping oligonucleotides and subsequently cloned under control of the lactococcal promoter P59 (Van der Vossen et al., 1987) in pNuc9 as described previously (Van de Guchte et al., 1998).

RNA manipulations. For RNA extraction, cultures were grown in the presence of Trp, Asn and erythromycin to an $\mathrm{OD}_{600}$ of $0 \cdot 6$. The cells were collected by centrifugation and resuspended in an equal volume of fresh medium containing or lacking Asn or Trp. After incubation for $30 \mathrm{~min}$ in these media at $30^{\circ} \mathrm{C}$, the cells were harvested and RNA was extracted as described by Raya et al. (1998). Aliquots of $20 \mu \mathrm{g}$ were subjected to electrophoresis in $1 \%$ agarose and blotted onto Nytran membranes (NY12N; Schleicher \& Schuell). Northern 
Table 1. Bacterial strains and plasmids

\begin{tabular}{|c|c|c|}
\hline $\begin{array}{l}\text { Bacterial strain } \\
\text { or plasmid }\end{array}$ & Relevant features & Source or reference \\
\hline \multicolumn{3}{|c|}{ L. lactis subsp. lactis } \\
\hline IL56012 & $\begin{array}{l}\text { IL1403 containing a promoterless E. coli lacZ } \\
\text { gene on the chromosome }\end{array}$ & Van de Guchte et al. (1998) \\
\hline IL56013 & $\begin{array}{l}\text { IL1403 containing a trpE-lacZ fusion on the } \\
\text { chromosome; specifier TGG (Trp) }\end{array}$ & Van de Guchte et al. (1998) \\
\hline IL56016 & $\begin{array}{l}\text { IL1403 containing a } \operatorname{trpE} \text {-lacZ fusion on the } \\
\text { chromosome; specifier AAC (Asn) }\end{array}$ & Van de Guchte et al. (1998) \\
\hline IL56026 & $\begin{array}{l}\text { IL1403 containing a trpE-lacZ fusion on the } \\
\text { chromosome; specifier AAC (Asn), mutation } \\
\text { ML1 (Fig. 4b) }\end{array}$ & This study \\
\hline IL56027 & $\begin{array}{l}\text { IL1403 containing a trpE-lacZ fusion on the } \\
\text { chromosome; specifier AAC (Asn), mutation } \\
\text { ML2 (Fig. 4b) }\end{array}$ & This study \\
\hline \multicolumn{3}{|l|}{ Plasmids } \\
\hline pIL931 & Chromosomal delivery vector & Van de Guchte et al. (1998) \\
\hline pIL253 & $\mathrm{Em}^{\mathrm{r}}, \mathrm{pAM} \beta$-derived cloning vector & Simon \& Chopin (1988) \\
\hline pNuc9 & $\begin{array}{l}\mathrm{Em}^{\mathrm{r}} \text {, pIL253 derivative containing the strong } \\
\text { lactococcal promoter P59 }\end{array}$ & Le Loir et al. (1998) \\
\hline pIL938 & $\mathrm{Em}^{\mathrm{r}}, \mathrm{pNuc} 9$ derivative specifying $\mathrm{tRNA} \mathrm{Arp}^{\mathrm{Trp}}$ & Van de Guchte et al. (1998) \\
\hline pIL939 & $\mathrm{Em}^{\mathrm{r}}, \mathrm{pNuc9}$ derivative specifying tRNA ${ }^{\mathrm{Asn}}$ & Van de Guchte et al. (1998) \\
\hline pIL940 & $\begin{array}{l}\text { Em }^{\mathrm{r}} \text {, pNuc9 derivative specifying hybrid tRNA } \\
1 \text { (Fig. 5) }\end{array}$ & This study \\
\hline pIL941 & $\begin{array}{l}\text { Em }^{\mathrm{r}} \text {, pNuc9 derivative specifying hybrid tRNA } \\
2 \text { (Fig. 5) }\end{array}$ & This study \\
\hline pIL942 & $\begin{array}{l}\text { Em }^{\mathrm{r}} \text {, pNuc9 derivative specifying hybrid tRNA } \\
3 \text { (Fig. 5) }\end{array}$ & This study \\
\hline pIL943 & $\begin{array}{l}\text { Em }^{\mathrm{r}} \text {, pNuc9 derivative specifying hybrid tRNA } \\
4 \text { (Fig. 5) }\end{array}$ & This study \\
\hline pIL944 & $\begin{array}{l}\mathrm{Em}^{\mathrm{r}} \text {, pNuc9 derivative specifying hybrid tRNA } \\
5 \text { (Fig. 5) }\end{array}$ & This study \\
\hline pIL945 & $\begin{array}{l}\text { Em }^{\mathrm{r}} \text {, pNuc9 derivative specifying hybrid tRNA } \\
6 \text { (Fig. 5) }\end{array}$ & This study \\
\hline pIL946 & $\begin{array}{l}\text { Em }^{\mathrm{r}} \text {, pNuc9 derivative specifying hybrid tRNA } \\
7 \text { (Fig. 5) }\end{array}$ & This study \\
\hline pIL947 & $\begin{array}{l}\text { Em }^{\mathrm{r}} \text {, pNuc9 derivative specifying hybrid tRNA } \\
8 \text { (Fig. 5) }\end{array}$ & This study \\
\hline
\end{tabular}

hybridizations were performed using either of the following $\gamma$ ${ }^{32} \mathrm{P}$ labelled oligonucleotide probes: 5'-CTGGTCACTTCGATGGTT-3' (complementary to the E. coli lacZ gene) or 5'GGCGTATGTCACGCGGTGCCACCG-3' (complementary to lactococcal trp leader transcripts). Transcripts were quantified using a PhosphorImager (Storm; Molecular Dynamics). The relative readthrough was determined as described previously (Van de Guchte et al., 1998). Briefly, the relative amount of full-length transcript was determined using a lacZspecific probe and a correction for the Trp-independent variation in the level of transcription initiation (Raya et al., 1998) and possible gel charging errors was made after rehybridization with a $t r p$ leader-specific probe. Comparison of the signals obtained with the $l a c Z$ probe for different lanes on the same Northern blot allowed, after correction based on the signals obtained with the $\operatorname{trp}$ leader probe, calculation of a relative antitermination value for each lane (relative to a given lane on the same blot). The presented relative antitermination values are mean values from two to five experiments.

\section{RESULTS}

\section{Specifier-codon-independent transcription antitermination}

The isogenic L. lactis strains IL56013 and IL56016 carry, in addition to the $\operatorname{trp}$ operon, a $\operatorname{trpE}-\mathrm{lac} Z$ fusion on the chromosome, inserted in the transcriptionally silent $h$ is operon (Fig. 2; Van de Guchte et al., 1998). Upstream of the translational fusion between $\operatorname{trp} E$, the first gene of the $t r p$ operon, and lac $Z$ the $t r p$ promoter and leader sequence are present. In IL56013, this leader sequence is identical to the wild-type leader of the trp operon, whereas in IL56016 the leader sequence of the trpE-lacZ fusion contains the specifier codon AAC (Asn) instead of TGG (Trp).

Earlier studies (Van de Guchte et al., 1998) showed that transcription antitermination in the trpE-lacZ fusion 
(a)

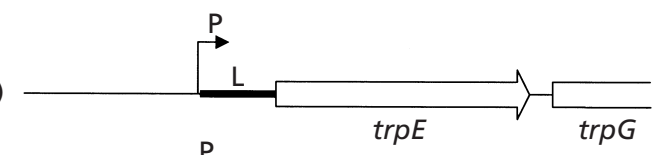

(b)

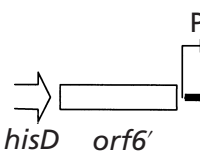

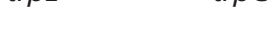
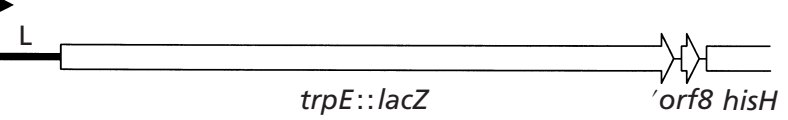

Fig. 2. trp leader sequences on the chromosomes of IL56013 and IL56016. trp promoter ( $\mathrm{P}$ ) and leader (L) sequences are present in the trp operon (a) and in a trpElacZ fusion located in the his operon (b). In IL56013 both leader sequences are identical (wild-type). In IL56016 the leader sequence of the trpE-lacZ fusion contains the specifier codon AAC (Asn) instead of TGG (Trp).

(a)

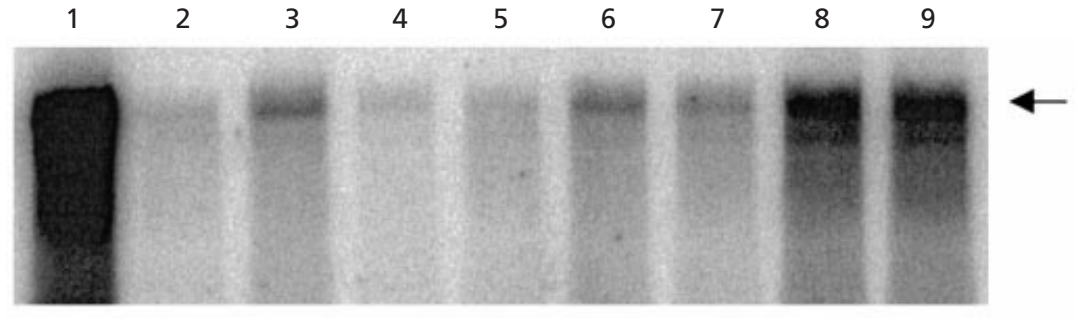

(b)

Specifier

tRNA

CDM

Antitermination

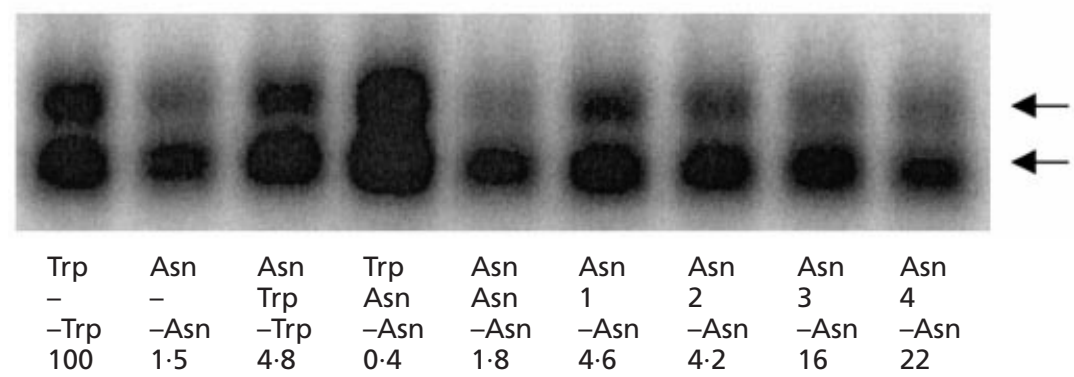

Fig. 3. Effect of native and chimeric tRNAs on transcription antitermination in the trpE-lacZ fusion. RNA was isolated from $L$. lactis cells after a growth medium shift as indicated in Methods and used to prepare a Northern blot that was hybridized with a lacZ-specific probe to visualize full length trpE-lacZ transcripts (a) and with a trp leader-specific probe to visualize the $290 \mathrm{bp}$ trp leader transcript and its $160 \mathrm{bp}$ processing product (Raya et al., 1998) (b). The trpE-lacZ transcript indicated by the arrow in (a) has an estimated size of $8 \mathrm{~kb}$, corresponding to the size expected from a transcript initiated at the $P_{\text {trp }}$ promoter and terminated at the his transcription terminator. Specifier, specifier codon present in the trp leader upstream of the chromosomal trpE-lacZ fusion: Asn (AAC) or Trp (TGG). tRNA, overexpressed tRNAs are indicated; numbers refer to chimeric tRNAs described in Fig. 5. Strains in which no tRNA is overexpressed contain plasmid plL253. Amino acids lacking from the CDM after the growth medium shift are indicated. Relative antitermination was determined as described in Methods. Antitermination in the wild-type trpE-lacZ fusion under conditions of Trp starvation is arbitrarily set to 100 .

with the wild-type leader was strongly induced by the absence of Trp from the culture medium (Fig. 3, lane 1), whereas the specifier (AAC) mutant was induced at only a very low level by Asn depletion (Fig. 3, lane 2). However, under conditions of overexpression of the lactococcal tRNA ${ }^{\operatorname{Trp}}$ in the specifier (AAC) mutant, it became evident that induction was still possible, albeit at a low level, by Trp depletion (Fig. 3, lane 3). In contrast, the wild-type fusion could not be induced by Asn depletion under conditions of overexpression of tRNA $^{\text {Asn }}$ (Fig. 3, lane 4).

These results indicate that transcription antitermination induced by Trp depletion in a specifier mutant may involve specifier-codon-independent interactions between $\mathrm{tRNA}^{\operatorname{Trp}}$ and the $\operatorname{trp}$ leader transcript. This type of interaction did not seem to occur between tRNA $^{\mathrm{Asn}}$ and the $\operatorname{trp}$ leader.

\section{Sequence complementarity between tRNA ${ }^{\mathrm{Trp}}$ and the trp leader transcript}

The above observations prompted a renewed analysis of the $\operatorname{trp}$ leader. The lactococcal tRNA ${ }^{\mathrm{Trp}}$ sequence, which has recently become available (P. Renault, unpublished), was used to search for mRNA-tRNA complementarity that could be indicative of additional recognition mechanisms. As shown in Fig. 4(a), there is extensive sequence complementarity between the trp leader and tRNA ${ }^{\text {Trp }}$. A stretch of 21 nt in stem-loop structure IV of the $\operatorname{trp}$ leader (Fig. 1) is complementary to the D-arm and the anticodon arm of tRNA ${ }^{\mathrm{Trp}}$, whereas another stretch of $18 \mathrm{nt}$ is complementary to the T-arm. Moreover, parts of the latter sequence are repeated several times upstream of the $18 \mathrm{nt}$ sequence in stem-loop structure II of the $\operatorname{trp}$ leader (Fig. 1). This large extent of mRNA-tRNA complementarity suggests a functional 

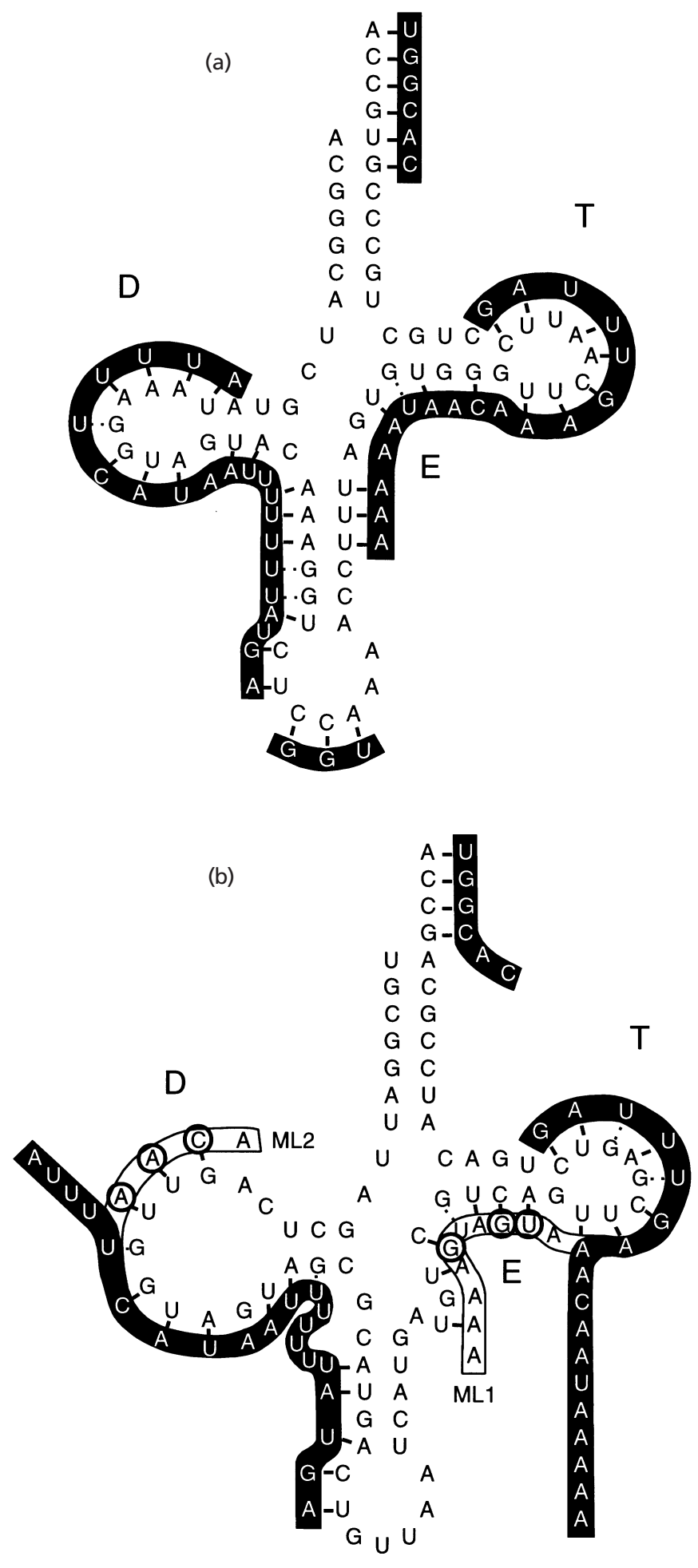

Fig. 4. Complementarity between trp leader fragments and (a) tRNA $^{\text {Trp }}$ or (b) tRNA ${ }^{\text {Asn }}$. D, tRNA D-arm; T, tRNA T-arm; E, tRNA extra arm. trp leader sequence fragments complementary to tRNA $^{\text {Trp }}$ are highlighted (clockwise: anti-acceptor, T-complementary region, specifier codon, D-complementary region); compare with Fig. 1 for the localization of these fragments. Boxed sequences ML1 and ML2 represent mutated trp leader sequences; mutations (encircled nucleotides) are introduced to improve the complementarity to tRNA ${ }^{\text {Asn }}$. role for stem-loop structures II and IV in the establishment or maintenance of the mRNA-tRNA interaction. If these complementary regions were important for an efficient mRNA-tRNA interaction, it would be expected that tRNA ${ }^{\mathrm{Asn}}$ would show less, or differently structured, complementarity to the trp leader than $\mathrm{tRNA}^{\mathrm{Tr}}$, since the specifier (AAC) mutant showed only a weak response to Asn depletion. As shown in Fig. 4(b), tRNA Asn (P. Renault, unpublished) does indeed show substantially lower complementarity than tRNA ${ }^{\operatorname{Trp}}$ to the $\operatorname{tr} p$ leader. In addition, tRNA ${ }^{\mathrm{Asn}}$ differs markedly from $t \mathrm{RNA}^{\mathrm{Trp}}$ in the size of its D-stem, D-loop and extra arm.

\section{Effect of chimeric tRNAs on transcription antitermination in the specifier Asn mutant}

To examine whether the above differences between tRNA ${ }^{\mathrm{Asn}}$ and $\mathrm{tRNA}^{\mathrm{Trp}}$ could be responsible for the weak antitermination response in the specifier (AAC) mutant, chimeric tRNAs were constructed. Based on the L. lactis tRNA ${ }^{\mathrm{Asn}}$ gene, recombinant genes were created in which (i) the D-arm, (ii) the T-arm and extra arm or (iii) both sequences were replaced by the corresponding arms of tRNA ${ }^{\text {Trp }}$ (Fig. 5). In addition, a gene was created based on the tRNA ${ }^{\operatorname{Trp}}$ gene in which the anticodon CCA (anti-TGG, Trp) was replaced by GTT (anti-AAC, Asn). The resulting tRNA can be regarded as a tRNA ${ }^{A s n}$ in which all but the anticodon is replaced by the corresponding parts of tRNA ${ }^{\text {Trp }}$. The different chimeric tRNAs all contain the identity elements of tRNA $^{\mathrm{Asn}}$, the anticodon GUU and the discriminator base $G$, and therefore are likely to be chargeable by asparaginyl-tRNA synthetase, even if tRNA ${ }^{\text {Trp }}$ and tRNA $^{\text {Asn }}$ are normally charged by synthetases that belong to two different classes (Giegé et al., 1998).

tRNA $^{\text {Asn }}$ and the chimeric tRNAs were overproduced in the specifier (AAC) mutant and their effect on transcription antitermination in the trpE-lacZ fusion was studied. Under conditions of Asn limitation, the overproduction of tRNA ${ }^{\text {Asn }}$ affected antitermination only a little or not at all (Fig. 3, lanes 2 and 5). In contrast, overproduction of tRNA ${ }^{\mathrm{Asn}}$ containing either the D-arm or the T-arm and extra arm of tRNA ${ }^{\text {Trp }}$, resulted in a clearly enhanced level of antitermination which was increased even further when all these tRNA ${ }^{\operatorname{Trp}}$ arms were present (Fig. 3, lanes 6, 7, 8). Expression of the latter chimera resulted in a level of antitermination approaching that realized by the overproduction of tRNA $^{\operatorname{Trp}}$ in which the anticodon had been replaced by the anticodon GUU (anti-AAC, Asn) (Fig. 3, lane 9). These results suggest an important role for the tRNA $\mathrm{D}$ - and $\mathrm{T}$-arms in the mRNA-tRNA interaction.

Since it is the ratio between the amount of uncharged and charged tRNA that determines whether, and to what extent, antitermination occurs (Grundy et al., 1994; Putzer et al., 1995; Van de Guchte et al., 1998), the possibility remained that the increased antitermination in the presence of chimeric tRNAs was due to an 


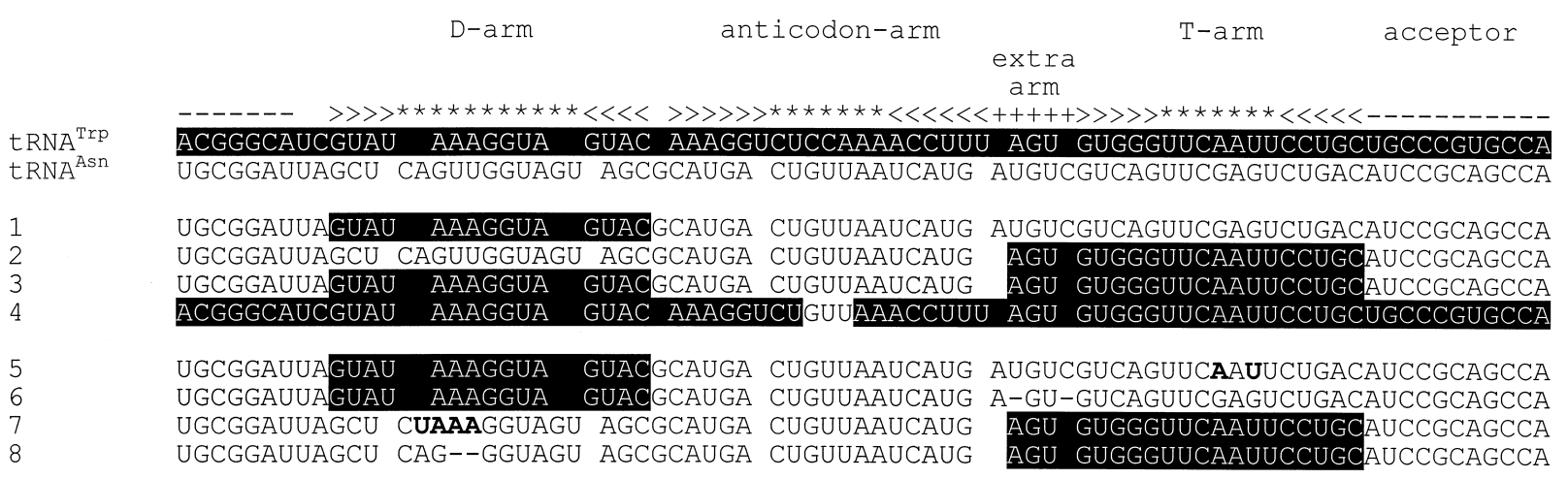

Fig. 5. Sequence of wild-type and chimeric tRNAs. 1, tRNA Asn in which the D-arm has been replaced by the D-arm of tRNA ${ }^{\text {Trp }} ; 2$, tRNA ${ }^{A s n}$ in which the T-arm and the extra arm have been replaced by the T-arm and the extra arm of tRNA ${ }^{\text {Trp }}$; 3 , tRNA ${ }^{A s n}$ in which the D-arm, the T-arm and the extra arm have been replaced by the D-arm, the T-arm and the extra arm of tRNA ${ }^{\text {Trp }} ; 4$, tRNA ${ }^{A s n}$ in which the whole sequence except for the anticodon has been replaced by the sequence of tRNA $^{\text {Trp }}$, i.e. tRNA ${ }^{\text {Trp }}$ with anticodon Asn; 5 , tRNA ${ }^{A s n}$ in which the D-arm has been replaced by the D-arm of tRNA ${ }^{\operatorname{Trp}}$ (tRNA 1), with 2 nt substitutions in the T-loop (bold type); 6 , tRNA ${ }^{A s n}$ in which the D-arm has been replaced by the D-arm of tRNA $^{\text {Trp }}$ (tRNA 1), with a 2 nt size reduction of the extra arm $(-) ; 7$, tRNA $^{A s n}$ in which the T-arm and the extra arm have been replaced by the T-arm and the extra arm of tRNA Trp (tRNA 2), with 4 nt substitutions in the D-loop (bold type); 8 , tRNA $^{\text {Asn }}$ in which the T-arm and the extra arm have been replaced by the T-arm and the extra arm of tRNA ${ }^{\text {Trp }}$ (tRNA 2), with a 2 nt size reduction of the D-loop (-). RRNA $^{\text {Trp }}$, tRNA ${ }^{A s n}$, wild-type sequences of $L$. lactis subsp. lactis; ---, >>>, $<<<$, stem; ${ }^{* *}$, loop.

Table 2. Transcription antitermination in the presence of an excess of Asn

\begin{tabular}{|lclc|}
\hline Specifier* & tRNA $\dagger$ & CDM & Antitermination $\$$ \\
\hline Trp & - & - Trp & 100 \\
Asn & - & Complete & $0 \cdot 2$ \\
Asn & Asn & Complete & $0 \cdot 2$ \\
Asn & 1 & Complete & $0 \cdot 2$ \\
Asn & 2 & Complete & $0 \cdot 2$ \\
Asn & 3 & Complete & $3 \cdot 7$ \\
Asn & 4 & Complete & $4 \cdot 5$ \\
\hline
\end{tabular}

*Specifier codon present in the trp leader upstream of the chromosomal trpE-lacZ fusion: Asn (AAC) or Trp (TGG).

† Overexpressed tRNAs are indicated. Numbers refer to chimeric tRNAs described in Fig. 5. Strains in which no tRNA is overexpressed contain plasmid pIL253.

$\ddagger-$ Trp indicates that Trp was lacking from the medium after the growth medium shift.

Relative antitermination determined as described in Methods. Antitermination in the wild-type trpE-lacZ fusion under conditions of Trp starvation is arbitrarily set to 100 .

impaired chargeability of these tRNAs. To rule out this possibility, the experiments were repeated in the presence of an excess of Asn in the culture medium. Under these conditions, substitution of the D- or T-arm and extra arm in tRNA ${ }^{\text {Asn }}$ did not induce antitermination (Table 2, compare chimeric tRNAs 1 and 2 to tRNA ${ }^{\mathrm{Asn}}$ ), indicating that these chimeric tRNAs can be charged efficiently and that enhanced antitermination during Asn starvation using these tRNAs thus results from an improved interaction with the trp leader. The over- expression of $\mathrm{tRNA}^{\mathrm{Asn}}$ in which both the D-arm and the $\mathrm{T}$-arm were substituted, or tRNA ${ }^{\mathrm{Trp}}$ with anticodon Asn resulted in antitermination to approximately $20 \%$ of the level obtained under conditions of Asn limitation (compare chimeric tRNAs 3 and 4 in Table 2 to the same tRNAs in Fig. 3). This result indicates that these chimeric tRNAs can also be charged with Asn, albeit with reduced efficiency. The enhanced antitermination using these tRNAs thus appears to be due essentially to an improved interaction with the trp leader.

Together, these results strongly suggest that the chimeric tRNAs enhance antitermination through an improved interaction with the leader transcript and, consequently, that the tRNA D- and T-arms play a role in mRNAtRNA recognition.

\section{Effect of mutations in chimeric tRNAs on transcription antitermination}

The lactococcal tRNA ${ }^{\text {Trp }}$ and $\mathrm{tRNA}^{\mathrm{Asn}}$, and the chimeric tRNAs not only differ in the nucleotide sequences of their D-, T- and extra arms, but also in the sizes of the D-stem and D-loop, as well as the size of the extra arm, features that may also affect mRNA-tRNA interaction. To address the question whether the antitermination-enhancing effects of the chimeric tRNAs could be ascribed to either an improved mRNAtRNA sequence complementarity or to an enhanced structural compatibility because of the sizes of the Dand extra arm, the effects of small mutations in tRNA ${ }^{\text {Asn }}$ were studied.

To allow for maximum interaction between mRNA and tRNA, the chimeric tRNA containing the D-arm of $\mathrm{tRNA}^{\text {Trp }}$ was used to create mutations in the T-arm or 
Table 3. Effect of mutations in chimeric tRNAs on transcription antitermination

Overexpressed tRNAs are indicated. tRNA numbers refer to chimeric tRNAs described in Fig. 5. Antitermination values, relative to tRNA 1 for tRNAs 5 and 6 , and relative to tRNA 2 for tRNAs 7 and 8, were determined as described in Methods under conditions of Asn starvation in the trpE-lacZ fusion with specifier codon AAC (Asn). Antitermination values for tRNAs 1 and 2 are arbitrarily set to 100 .

\begin{tabular}{|lccc|}
\hline tRNA & Antitermination & tRNA & Antitermination \\
\hline 1 & 100 & 2 & 100 \\
5 & 89 & 7 & 95 \\
6 & 135 & 8 & 69 \\
\hline
\end{tabular}

in the extra arm, whereas the chimeric tRNA containing the T-arm of tRNA ${ }^{\operatorname{Trp}}$ was used to create mutations in the $\mathrm{D}$-arm. The resulting tRNAs are shown in Fig. 5. In tRNAs 5 and 7 the complementarity of the T- or the Darm, respectively, to the trp leader has been improved, without changing the size of these arms or the extra arm. In tRNA 6 the size of the extra arm has been reduced, thereby also slightly improving the complementarity to the $\operatorname{trp}$ leader. In tRNA 8 the size of the D-loop has been reduced without improving mRNA complementarity.

The results of overproduction of these modified tRNAs, presented in Table 3, show that it was not possible to separate sequence complementarity and arm size effects. Only the overexpression of tRNA 6 resulted in a slightly enhanced antitermination. These results suggest that a combination of primary sequence and structure information is responsible for the correct interaction of mRNA and tRNA.

\section{Effect of mutations in the trp leader on transcription antitermination}

In a second approach, complementary to the one described above, mutations were made in the trp leader to enhance its complementarity to either the T-arm (ML1) or the D-arm (ML2) of tRNA ${ }^{\mathrm{Asn}}$. The mutations are indicated in Fig. 4(b). In the strain carrying the mutation aimed at an improved interaction with the Darm of $\mathrm{tRNA}^{\mathrm{Asn}}$, the hybrid tRNA containing the T-arm of tRNA $^{\text {Trp }}$ was overexpressed, and in the strain carrying the mutation aiming at an improved interaction with the T-arm of tRNA ${ }^{\text {Asn }}$, the hybrid tRNA containing the D-arm of tRNA ${ }^{\text {Trp }}$ was overexpressed, to allow for maximum interaction between mRNA and tRNA. Under conditions of Asn limitation, neither of these mutations gave rise to an enhanced level of antitermination $[19 \%$ of the antitermination in the wild-type leader with anticodon AAC (Asn) for ML1; $74 \%$ for ML2]. This result is in agreement with the results obtained with mutations in the chimeric tRNAs described above, where improved sequence complementarity alone did not suffice to enhance antitermination, probably due to the fact that structural incompatibilities between tRNA and mRNA remain.

\section{mRNA-tRNA complementarity in analogous systems}

On the basis of the conserved structural features of their leader transcripts, several aminoacyl-tRNA synthetase genes and amino acid biosynthesis operons in Bacillus subtilis and L. lactis have been postulated to be regulated by tRNA-mediated transcription antitermination (Grundy \& Henkin, 1993, 1994; Henkin, 1994; Van de Guchte et al., 1998; Luo et al., 1998; Chopin et al., 1998; Delorme et al., 1999). Taking the L. lactis trp leader as a model, we looked for the presence of mRNA-tRNA sequence complementarity in the systems listed in Fig. 6, focusing on complementarity between stem-loop structure II of the leader transcript and the Tarm of the cognate tRNA and between stem-loop structure IV and the D-arm of the tRNA.

The results presented in Fig. 6 show that while the extent of the complementarity in the L. lactis trp leader seems to be exceptional, in all cases short stretches of complementarity between the respective mRNA and tRNA structures could be found. These sequences comprise at least three and generally from four to nine consecutive complementary nucleotides (including G: $\mathrm{U}$ pairs). Interestingly, nearly all involve at least a part of the single-stranded loop regions in the tRNAs, in the majority of cases complementary to single-stranded loop regions in the leader transcripts. In a number of cases complementarity to the tRNA T-arm involves the single-stranded spacer region between stem-loop structures II and III of the leader transcripts, like in the L. lactis trp leader. In some cases this complementary sequence extends into stem-loop structure III of the leader transcript.

\section{DISCUSSION}

Whereas various studies have highlighted the conservation of structures and short, functionally important, conserved sequence elements at various positions in the leader transcripts that are subject to tRNA-mediated transcription antitermination (Rollins et al., 1997; Chopin et al., 1998; Luo et al., 1998), the present study emphasizes the differences between the different systems that would contribute to the specificity of the antitermination response. The specifier codon has been recognized as a major determinant of specificity (Grundy \& Henkin, 1993), while Grundy et al. (1994) showed that an anti-acceptor nucleotide complementary to the tRNA discriminator base also contributes to tRNA recognition. However, as exemplified by the low inducibility of antitermination in the L. lactis trp leader with specifier codon AAC (Asn) (Van de Guchte et al., 1998) codon-anticodon and acceptor-anti-acceptor correspondence are not sufficient for an efficient mRNAtRNA interaction.

In this specifier mutant of the lactococcal trp leader, antitermination can still be induced by Trp limitation, 
\begin{tabular}{|l|l|l|l|l|l}
\hline Acceptor D-arm anticodon-arm extra arm T-arm acceptor \\
\hline
\end{tabular}

a 5 '-ACGGGCAUCGUAUAAAGGUAGUACAAAGGU-CUCCAAAACCUUUAGUGUGGGUUCAAUUCCUGCUGCCCGUGCCA-3 '

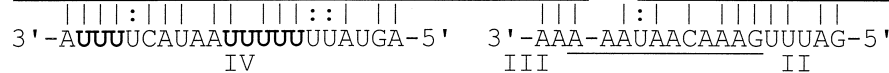

b 5 ' -GGCGGAUGUGGUGAAGUGGUUAACACACCGGCUUGUGGCGCCGGCACUCGCGAGUUC GAUCCUCGUCAUCCGCC- 3 '

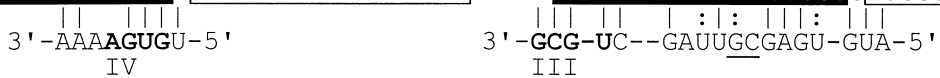

15 ' -GGAGAGCUGUCCGAGUGGUCGAAGGAGCACGAUUGGAAAUCGUGUAGGCGGUCAACUCCGUCUCAAGGGUUCGAAUCCCUUGCUCUCCGCCA-3 ' $::||:|1|:$
$3^{\prime}-$ UUAGUUUCUं-5
IV $3^{\prime}$ - UUUACGGA-UGAG-5'

25 '-GGCGGCAUAGCCAAGUG-GUAAGGCAGAGGUCUGCAAAACCUUUAUCCCCGGUUCGAAUCCGGGUGUCGCCU-3 '

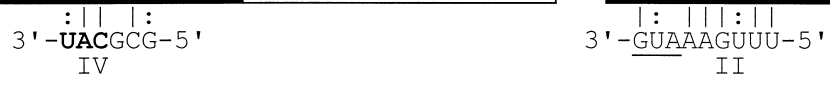

35 ' -CGGGAAGUAGCUCAGCUUGGUAGAGCACAUGGUUUGGGACCAUGGGGUCGCAGGUUCGAAUCCUGUUUUCCCGACCA-3 '

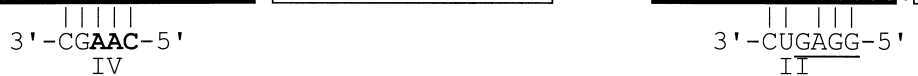

3b 5' -CGGGAAGUAGCUCAG--CUUGGUAGAGCACAUGGUUUGGGACCAUGGGGUCGCAGGUUCGAAUCCUGUCUUCCCGACCA-3'

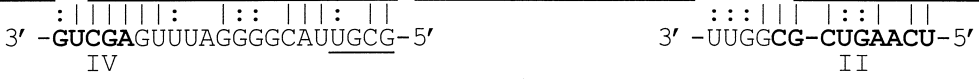

45 '-GGGCCUGUAGCUCAGCUGGUUAGAGCGCACGCCUGAUAAGCGUGAGGUCGGUGGUUCGAGUCCACUCAGGCCCA - 3 '

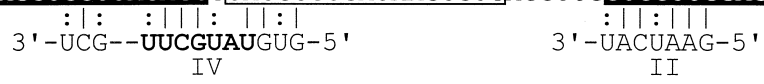

5a 5 ' -GGAGGGGUAGCGAAGUGCUAAACGCGGCGGACUGUAAAUCCGCUCCCUCAGGGUUCGG--CAG-UUCGAAUCUGCCCCCCUCCA-3 '

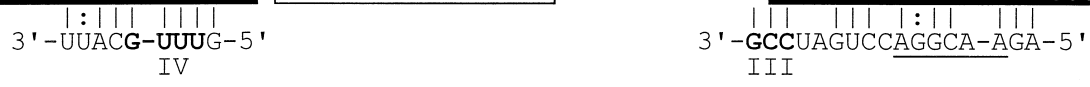

5b 5 ' -GGAGGGGUAGCGAAGUGCUAAACGCGGCGGACUGUAAAUCCGCUCCCUCAGGGUUCGGCAGUUCG-AAUCUGCCCCCCUCCA-3 '

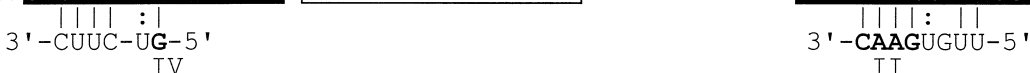

$$
\text { IV II }
$$

65 '-GGAGGAUUAGCUCAGCUGGGAGAGCA_UCUGCCUUACAAGCAGAGGGUCGGCGGUUCGAGCCCGUCAUCCUCCACCA-3 '

$$
\begin{gathered}
|:| 1 \mid:: \\
\text { 3'-UUGAGUU-5' } \\
\text { 3'-AAGUUC-5' }
\end{gathered}
$$

75 ' -GGCUCGGUAGCUCA-GUUGGUAGAGCA ACGGACUGAAAAUCCGU-GUGUCGGCGGUUCGAUUCCGUCCCGAGCCACCA-3'

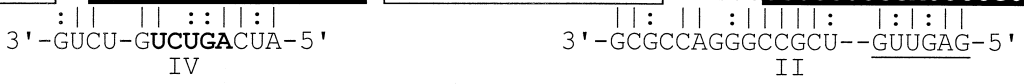

8a 5 ' -GCUUCCAUAGCUC-AGCA-GGUAGAGCACUUCCAUGGUAAGGAAGAGGUCAGCGGUUCGAG--CCCGCUUGGAAGCU-3 '

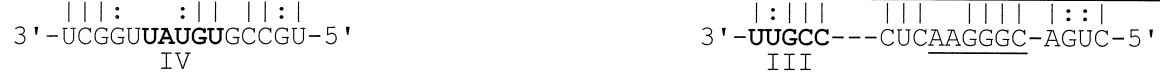

8b 5 ' -GCUUCCAUAGCUCAGCAGGUAGAGCACUUCCAUGGUAAGGAAGAGGUCAGCGGUUCGAG--CCCGCUUGGAAGCU- 3 '

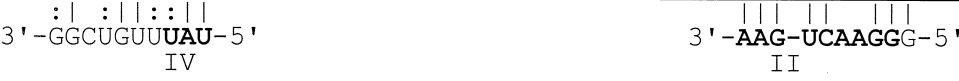

8c 5 ' -GCUUCCAUAGCUCAGCAG--GUAGAGCA CUUCCAUGGUAAGGAAGAGGUCAGCGGUUCGAGCCCGCUUGGAAGCU-3 -

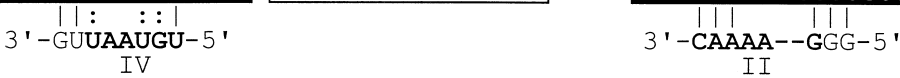

$9 a \quad 5$ '-GCGGUCGUGGCGGAAUGGCAGACGCGCUAGGUUGAGGGCCUAGUGGGUGAAUAACCCGUGGAGGUUCAAGUCCU-CUCGGCCGCA-3 '

$$
\begin{aligned}
& \begin{array}{c}
:||:|||| \\
3^{\prime} \text {-UUAGCCG-5' } \\
\text { IV }
\end{array}
\end{aligned}
$$

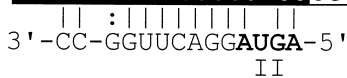

9b 5 ' -GCGGUCGUGGCGGAAUGGCAGACG-CGCUAGGUUGAGGGCCUAGUGGGUGAAUAACCCGUGGAGGUUCAAGUCCUCUCGGCCGCA-3 '

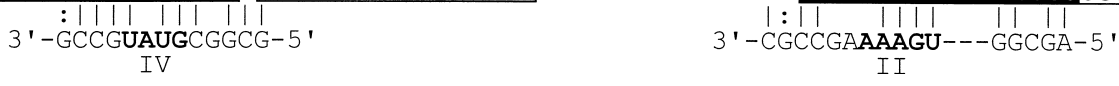

Fig. 6. For legend see facing page. 


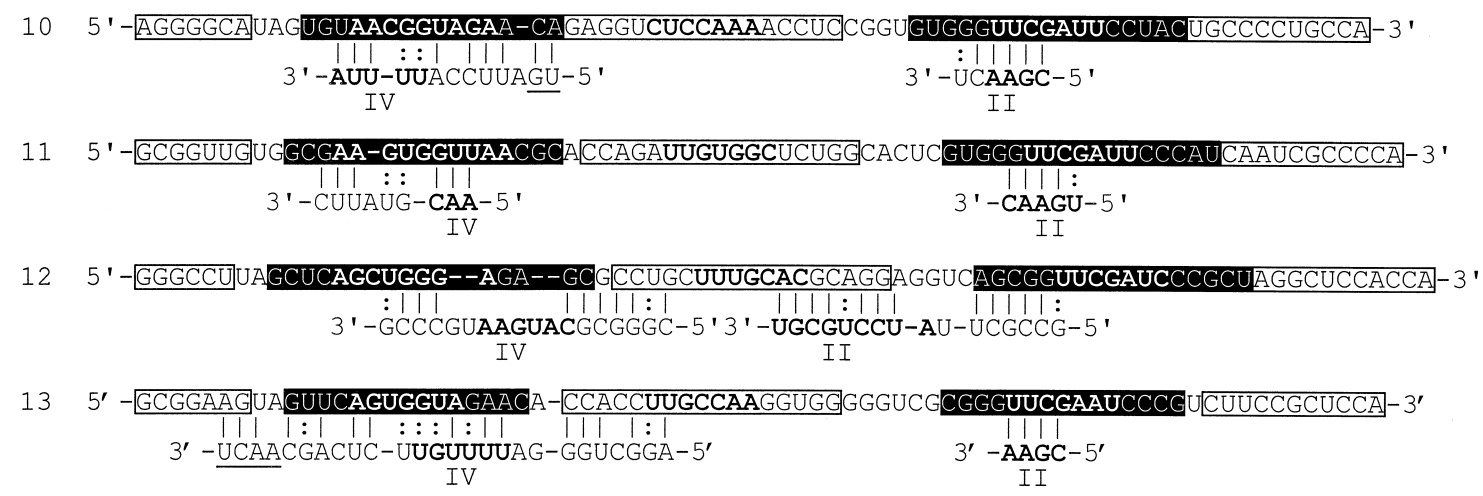

Fig. 6. Complementarity between tRNAs (upper line) and leader transcript fragments (lower line). Single-stranded loop regions are indicated by bold type. Single-stranded sequences between stem-loop structures in the leader transcripts are underlined. Roman numbers indicate stem-loop structures in leader transcripts analogous to the numbering in Fig. 1. tRNA/leader transcript: $a$, L. lactis tRNA ${ }^{\text {Trp }} / L$. lactis trp; $b$, L. lactis tRNA ${ }^{\text {His }} / L$. lactis his; 1 , B. subtilis tRNA ${ }^{\text {Ser }}$ (trnD-ser)/B. subtilis serS; $2, B$. subtilis tRNA Cys (trnD-cys)/B. subtilis cysES; $3, B$. subtilis tRNA ${ }^{\text {Pro }}$ (trnl-pro)/B. subtilis proBA; $3 \mathrm{~b}, B$. subtilis $\operatorname{tRNA}^{\text {Pro }}$ (trnB-pro)/B. subtilis proC; 4, B. subtilis tRNA ${ }^{l l e}(\operatorname{trnO}-i l e) / B$. subtilis ileS; 5a, B. subtilis tRNA ${ }^{\text {Tyr }}$ (trnSL-tyr1)/B. subtilis tyrS; $5 \mathrm{~b}, B$. subtilis tRNA ${ }^{\mathrm{Tyr}}\left(\operatorname{trnSL}-\right.$ tyr1)/B. subtilis tyrZ; $6, B$. subtilis tRNA ${ }^{\text {Val }}$ (trnJ-val)/B. subtilis valS; 7, B. subtilis tRNA $^{\text {Phe }}\left(\right.$ trnB-phe)/B. subtilis phes; 8a, B. subtilis tRNA ${ }^{\text {Thr }}\left(\right.$ trnl-thr)/B. subtilis thrS; 8b, B. subtilis tRNA ${ }^{\text {Thr }}$ (trnl-thr)/B. subtilis thrZ1; 8c, B. subtilis tRNA ${ }^{\text {Thr }}$ (trnl-thr)/B. subtilis thrZ2; 9a, B. subtilis tRNA ${ }^{\text {Leu }}$ (trnS-leu2)/B. subtilis ilv-leu; $9 \mathrm{~b}, B$. subtilis tRNA ${ }^{\text {Leu }}\left(\operatorname{trnS}\right.$-leu2)/B. subtilis leuS; 10, B. subtilis tRNA ${ }^{\operatorname{Trp}}\left(\operatorname{trn} D\right.$-trp)/B. subtilis trpS; 11, B. subtilis tRNA ${ }^{\text {His }}$ (trnDhis)/B. subtilis hisS; 12 , B. subtilis tRNA ${ }^{\text {Ala }}$ (trnA-ala)/B. subtilis alaS; 13, B. subtilis tRNA ${ }^{\text {Gly }}$ (trnD-gly)/B. subtilis glyQS.

suggesting that interactions between tRNA and leader transcript other than the codon-anticodon and acceptor-anti-acceptor interactions actively contribute to the stabilization of the antiterminator conformation of the leader transcript. As overexpression of lactococcal tRNA $^{\text {Asn }}$ under conditions of Asn limitation had no inducing effect in the wild-type trp leader, these interactions appear to be part of a recognition mechanism allowing the $\operatorname{trp}$ leader to specifically interact with tRNA ${ }^{\text {Trp }}$.

Asn starvation induced a low level of antitermination in the specifier (AAC) mutant. The overexpression of tRNA $^{\text {Asn }}$ did not affect this induction level. In contrast, the overexpression of chimeric tRNAs, consisting of tRNA $^{\text {Asn }}$ in which either the D-arm or the T-arm and extra arm were replaced by the corresponding sequences from tRNA ${ }^{\text {Trp }}$, gave rise to increased antitermination. The overexpression of a chimeric tRNA containing both sequences from tRNA ${ }^{\text {Trp }}$ augmented antitermination still further, as did the overexpression of $\mathrm{tRNA}^{\operatorname{Trp}}$ in which the anticodon had been replaced by the anti-Asn (anti-AAC) anticodon. The fact that in the single-arm replacement mutants antitermination was only observed in the absence of Asn from the culture medium indicates that these chimeric tRNAs can be charged efficiently and that, therefore, the enhanced antitermination is not due to an impaired charging of the tRNA, but to an intrinsic property of the replacement arm. In the multi-arm replacement mutants, enhanced antitermination largely depended on the absence of Asn, suggesting that here too the intrinsic arm properties rather than the partially impaired chargeability of these chimeric tRNAs are responsible for the enhancement.
Together, these results evoke an important role for the tRNA D- and T-arms in the specificity and efficacy of the mRNA-tRNA interaction. The exceptional degree of sequence complementarity between these tRNA arms and stem-loop structures IV and II, respectively, of the trp leader transcript strongly suggests the latter structures, to which until now no specific function had been assigned, as the counterparts in the interaction. The fact that mutations in $\mathrm{tRNA}^{\mathrm{Asn}}$ or the $\operatorname{trp}$ leader (with specifier Asn), aimed at improving the complementarity between the two sequences, did not result in enhanced antitermination shows that sequence complementarity per se is not sufficient to allow an efficient mRNA-tRNA interaction. Most likely, a combination of sequence and structural context is important. These observations lead us to propose a model of tRNAmediated transcription antitermination according to which the cognate tRNA would be recognized by the following 'identity elements' of the leader transcript: the specifier codon, the nucleotide complementary to the tRNA discriminator and stem-loop structures II and IV of the leader transcript.

The analysis of other leader transcripts from L. lactis and $B$. subtilis that are subject to tRNA-mediated antitermination showed the presence of short sequences that are complementary to the D- or T-arms of the respective cognate tRNAs in nearly all cases. Although none of these systems showed sequence complementarity as exhaustive as the lactococcal trp system, a general model of mRNA-tRNA recognition may hold true since in the majority of cases the mRNA-tRNA complementary sequences involve a number of nucleotides situated in loop structures or in other single- 
stranded regions of the respective RNAs. Scarabino et al. (1999) used an in vitro molecular selection approach to show that a tRNA preferentially binds RNA sequences complementary to its single-stranded anticodon, D- or T-loop sequence. The complementary sequences appeared to be present in a single-stranded loop or adjacent to a stem-loop structure in the selected RNAs, while the same sequences in an RNA molecule without secondary structure did not bind to the tRNA. Earlier studies by Grosjean et al. (1976) showed that complementary triplets in hairpin loops have a much higher affinity than free trinucleotides. In agreement with these results, long-range RNA-RNA interactions involved in E. coli ColEI replication control have been shown to be initiated by the interaction between complementary kissing loops of the folded RNA structures (Tomizawa, 1984). The proposed interactions between the tRNA D- and T-loops and stem-loop structures IV and II of the leader transcripts could fit a similar model in which very short complementary sequences placed in the correct structural context suffice for an interaction to take place.

Alternatively, one could conceive a model in which the nascent mRNA, after the establishment of a specifier codon-anticodon interaction, is guided in its folding by a dynamic interaction with the tRNA. In the lactococcal trp leader, the fact that parts of the $18 \mathrm{nt}$ sequence complementary to the tRNA ${ }^{\operatorname{Trp}} \mathrm{T}$-arm are repeated several times upstream of this sequence (Fig. 1) may be indicative of such a process.

In conclusion, our results clearly show the importance of the D- and T-arms of tRNA in the establishment or maintenance of an efficient, specific mRNA-tRNA interaction in the L. lactis trp leader. These tRNA sequences presumably interact with complementary sequences in the leader transcript, notably in stem-loop structures II and IV. Although the short tRNA-complementary sequences in the conserved stem-loop structures of other T-box leaders are less suggestive, these interactions may represent a general principle in tRNA-mediated transcription antitermination.

\section{ACKNOWLEDGEMENTS}

We thank P. Renault for critical reading of the manuscript.

\section{REFERENCES}

Bardowski, J., Ehrlich, S. D. \& Chopin, A. (1992). Tryptophan biosynthesis genes in Lactococcus lactis subsp. lactis. J Bacteriol 174, 6563-6570.

Birnboim, H. C. \& Doly, J. (1979). A rapid alkaline extraction procedure for screening recombinant plasmid DNA. Nucleic Acids Res 7, 1513-1523.

Chopin, A., Biaudet, V. \& Ehrlich, S. D. (1998). Analysis of the Bacillus subtilis genome sequence reveals nine new T-box leaders. Mol Microbiol 29, 662-664.
Delorme, C., Ehrlich, S. D. \& Renault, P. (1999). Regulation of expression of the Lactococcus lactis histidine operon. J Bacteriol 181, 2026-2037.

Dower, W. J., Miller, J. F. \& Ragsdale, C. W. (1988). High efficiency transformation of $E$. coli cells by high voltage electroporation. Nucleic Acids Res 16, 6127-6145.

Frenkiel, H., Bardowski, J., Ehrlich, S. D. \& Chopin, A. (1998). Transcription of the trp operon in Lactococcus lactis is controlled by antitermination in the leader region. Microbiology 144, 2103-2111.

Giegé, R., Sissler, M. \& Florentz, C. (1998). Universal rules and idiosyncratic features in tRNA identity. Nucleic Acids Res 26, 5017-5035.

Grosjean, H., Söll, D. G. \& Crothers, D. M. (1976). Studies of the complex between transfer RNAs with complementary anticodons. I. Origins of enhanced affinity between complementary triplets. J Mol Biol 103, 499-519.

Grundy, F. J. \& Henkin, T. M. (1993). tRNA as a positive regulator of transcription antitermination in B. subtilis. Cell 74, 475-482.

Grundy, F. J. \& Henkin, T. M. (1994). Conservation of a transcription antitermination mechanism in aminoacyl-tRNA synthetase and amino acid biosynthesis genes in Gram-positive bacteria. J Mol Biol 235, 798-804.

Grundy, F. J., Rollins, S. M. \& Henkin, T. M. (1994). Interaction between the acceptor end of tRNA and the T box stimulates antitermination in the Bacillus subtilis tyrS gene: a new role for the discriminator base. J Bacteriol 176, 4518-4526.

Henkin, T. M. (1994). tRNA-directed transcription antitermination. Mol Microbiol 13, 381-387.

Holo, H. \& Nes, I. F. (1995). Transformation of Lactococcus lactis by electroporation. Methods Mol Biol 47, 195-199.

Le Loir, Y., Gruss, A., Ehrlich, S. D. \& Langella, P. (1998). A nineresidue synthetic propeptide enhances secretion efficiency of heterologous proteins in Lactococcus lactis. J Bacteriol 180, 1895-1903.

Luo, D., Condon, C., Grunberg-Manago, M. \& Putzer, H. (1998). In vitro and in vivo secondary structure probing of the $t h r S$ leader in Bacillus subtilis. Nucleic Acids Res 26, 5379-5387.

Miller, J. H. (1972). Experiments in Molecular Genetics. Cold Spring Harbor, NY: Cold Spring Harbor Laboratory.

Putzer, H., Laalami, S., Brakhage, A. A., Condon, C. \& GrunbergManago, M. (1995). Aminoacyl-tRNA synthetase gene regulation in Bacillus subtilis: induction, repression and growth-rate regulation. Mol Microbiol 16, 709-718.

Raya, R., Bardowski, J., Andersen, P. S., Ehrlich, S. D. \& Chopin, A. (1998). Multiple transcriptional control of the Lactococcus lactis trp operon. J Bacteriol 180, 3174-3180.

Rollins, S. M., Grundy, F. J. \& Henkin, T. M. (1997). Analysis of cisacting sequence and structural elements required for antitermination of the Bacillus subtilis tyrS gene. Mol Microbiol 25, 411-421.

Scarabino, D., Crisari, A., Lorenzini, S., Williams, K. \& TocchiniValentini, G. P. (1999). tRNA prefers to kiss. $E M B O J \mathbf{1 8}$, 4571-4578.

Simon, D. \& Chopin, A. (1988). Construction of a vector plasmid family and its use for molecular cloning in Streptococcus lactis. Biochimie 70, 559-566.

Terzaghi, B. E. \& Sandine, W. E. (1975). Improved medium for lactic streptococci and their bacteriophages. Appl Microbiol 29, $807-813$. 
Tomizawa, J. (1984). Control of ColEI plasmid replication: the process of binding of RNA I to the primer transcript. Cell 38, 861-870.

Van de Guchte, M., Ehrlich, S. D. \& Chopin, A. (1998). tRNA ${ }^{\operatorname{Trp}}$ as a key element of antitermination in the Lactococcus lactis trp operon. Mol Microbiol 29, 61-74.
Van der Vossen, J. M. B. M., Van der Lelie, D. \& Venema, G. (1987). Isolation and characterization of Streptococcus cremoris WG2-specific promoters. Appl Environ Microbiol 53, 2452-2457.

Received 6 November 2000; revised 3 January 2001; accepted 16 January 2001. 